\title{
Investigación de un foco de leishmaniasis cutánea en la zona andina del departamento de Nariño *
}

\author{
César G. Arroyo ${ }^{1}$, Jorge Garzón ${ }^{2}$
}

\begin{abstract}
Resumen
En el municipio de Samaniego, ubicado en la zona andina del departamento de Nariño, se detectaron 14 casos de leismaniasis cutánea entre 1992 y 1995 , presentándose 13 de ellos (93\%) en niños menores de 13 años. En todos, las lesiones se localizaron en la cara. En tres de los casos se pudieron tipificar las cepas, correspondiendo a $L$. mexicana, resultado que coincide con la tipificación del primer paciente encontrado en Samaniego en 1984 y primer caso de leishmaniasis cutánea anérgica difusa informado en Colombia.

Paralelamente, se identificaron las especies de flebotomíneos recolectados en la zona, correspondiendo todos a Lutzomya columbiana (grupo verrucarum), hallazgo que concuerda con varios informes anteriores de la misma región desde 1940, cuando se buscaba el vector de la epidemia de bartonelosis en esta zona nariñense, hasta 1984 cuando el INS realizó muestreos a raíz del mencionado caso de leishmaniasis difusa encontrado por nosotros en ese municipio. Aunque no se logró demostrar infección natural en los 400 flebotomíneos capturados, sí se consiguió infectar algunos especímenes de Lutzomya columbiana, expuestos a hámsteres inoculados con la cepa de L. mexicana aislada de un paciente incluido en el estudio. Por este hallazgo y por la evidencia epidemiológica, se incrimina a Lutzomya columbiana como posible trasmisor de L. mexicana, vector que hasta la fecha no había sido involucrado en la trasmisión de leishmania.

Todos los pacientes estudiados respondieron al tratamiento convencional con glucantime $^{\circledR}$.
\end{abstract}

\section{Summary.}

Between 1992 and 1995, 14 cases of cutaneous leishmaniasis were detected in the municipality of Samaniego, located in the Nariño department, $13(93 \%)$ being presented amongst children aged less than 13 years of age. In all cases the lesions were localised on the face. In three cases it was possible to typify the strains as corresponding to $L$. mexicana, a result which coincided with the typification of the first patient to be found in Samaniego in 1984 and the first reported case in Colombia of diffuse anergic cutaneous leishmaniasis.

Parallelly, all sandfly species collected in the region were identified as belonging to Lutzomyia columbiana (verrucarum group), a finding which concurred with various pre-

Primer premio en el Segundo Congreso Nacional de Dermatología Pediátrica y Cuarto Seminario Internacional de Dermatología Pediátrica, Cali, noviembre de 1995

1 Dermatólogo, Coordinador del programa de leishmaniasis, Instituto Departamental de Salud de Nariño - IDSN, Pasto, Nariño.

2 Dermatólogo, IDSN 
vious reports from the same region since 1940 (the time of the search in this zone of Nariño for the bartonelosis epidemic vector) until 1984, when the INS carried out sampling as a result of the aforementioned case of diffuse leishmaniasis found by us in this municipality. Even though it was not possible to demonstrate natural infection in the 400 captive sandflies, some specimens of Lutzomyia columbiana were experimentally infected after being exposed to hamsters inoculated with an L. mexicana strain isolated from a patient included in this study. Because of this finding and from epidemiological evidence, Lutzomyia columbiana was incriminated as being the possible transmittor of $L$. mexicana, a vector which to date had not been involved in the transmission of leishmaniasis.

All patients studied responded to conventional treatment with Glucantime ${ }^{\circledR}$.

Las leishmaniasis son zoonosis que afectan la piel, las mucosas o las vísceras, resultantes del parasitismo por un protozoario al organismo, transmitido por la picadura de un insecto flebotomíneo (1). Las formas infectantes del parásito, o promastigotas, son flagelados que se desarrollan en el tubo digestivo del vector, un díptero del género Lutzomyia y, también, en medios de cultivo especiales. Una vez que son inoculados en la piel del hombre o del animal, invaden células histiocitarias y se transforman en amastigotas, formas inmóviles y redondeadas las cuales se reproducen activamente rompiendo el macrófago e invadiendo otros. Estas células parasitadas son fuente de infección para otros vectores con lo que se completa el ciclo biológico (2). La mayoría de las formas cutáneas y todas las mucocutáneas en el Nuevo Mundo, son producidas por géneros de Leishmania de los complejos brasiliensis y panamensis (3). Las formas cutáneas difusas son causadas por el complejo mexicano, que de acuerdo con la respuesta inmunológica del individuo pueden producir infección generalizada intratable, o reducirse a un número limitado de lesiones relativamente fáciles de controlar cuando el estado inmunológico es idóneo (3).

En Colombia, menos del $1 \%$ de los casos son causados por L. mexicana (2). La frecuencia de leishmaniasis cutánea en niños de 0-14 años corresponde aproximadamente a un $30 \%$ de los casos reportados en el país (1).

En el departamento de Nariño existen áreas endémicas de leishmaniasis cutánea y mucocutánea en el litoral Pacífico. Las especies del parásito aisladas en pacientes procedentes de esta zona corresponden a los complejos de $L$. brasiliensis y panamensis. No se ha encontrado hasta la fecha ningún caso de leishmaniasis visceral en el departamento (2).

En la región andina de Nariño, se han encontrado ocasionalmente casos aislados de leishmaniasis cutánea en los municipios de Samaniego, Sandoná, Consacá y Linares, ubicados en las estribaciones de la cordillera y cercanos a la hoya del río Guáitara (mapa 1).

Desde 1993, se han detectado en el municipio de Samaniego algunos casos autóctonos de leishmaniasis cutánea, la mayoría en niños y con lesiones localizadas en la cara. Su procedencia fue periurbana. Al examen parasitológico y en los cortes histopatológicos, se observó una gran cantidad de amastigotas del parásito.

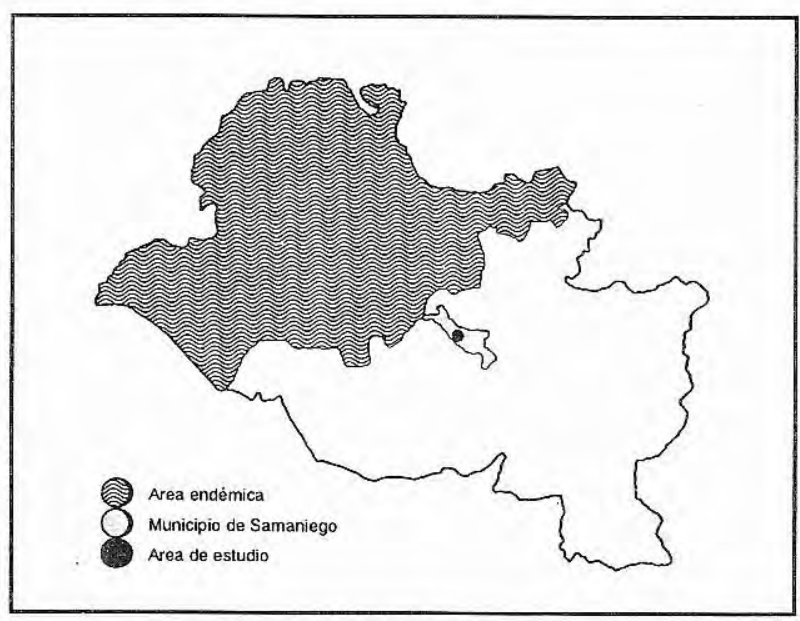

Mapa 1. Mapa epidemiológico del departamento de Nariño. 
Estas observaciones ya encontradas en el primer paciente de leishmaniasis cutánea anérgica difusa, diagnosticado en este municipio en 1984 (4), y que correspondió a una cepa de $L$. mexicana, nos llevaron a realizar el presente estudio, tendiente a demostrar si estos casos recientes eran provocados por la misma especie de leishmania.

El estudio se complementó con la identificación de las especies de Lutzomyia presentes en la zona, para establecer el probable vector de la cepa causal.

\section{Materiales y métodos}

El trabajo se realizó en el municipio de Samaniego, con una población de 28.740 habitantes y una extensión de $230 \mathrm{~km}^{2}$. Está situado en medio del Macizo Colombiano, aproximadamente a $100 \mathrm{~km}$ de la ciudad de Pasto, y hace parte de las poblaciones ubicadas a lo largo de la hoya del río Guáitara. Su altitud varia entre los 1.200 y 2.200 msnm. Su quebrada topográfica presenta condiciones climatológicas y ambientales que, de acuerdo con la clasificación de Holdgride, corresponde a "bosque húmedo premontano y bosque seco montano bajo" (5) .

Se conformó un grupo de trabajo constituido por dos médicos dermatológos como coordinadores, un bacteriólogo para el diagnóstico de laboratorio, un patólogo para el procesamiento y lectura de las biopsias y una enfermera como colaboradora en las diferentes actividades. Paralelamente, se organizó un equipo con los estudiantes de último año de biología de la Universidad de Nariño y personal del CIDEIM, con el fin de identificar las especies de flebotomíneos y los posibles reservorios presentes en el área de estudio.

Se realizó búsqueda activa de casos y se detectaron los casos de leishmaniasis cutánea autóctonos de esta zona, entre 1992 y 1995 (cuadro 1). Como criterios de inclusión, se determinaron los siguientes:

1) tener residencia permanente en el área de estudio;

2) no haber visitado áreas endémicas de leishmaniasis, y

Cuadro 1. Características de los pacientes estudiados.

\begin{tabular}{rcclccc}
\hline Casos & $\begin{array}{c}\text { Edad } \\
\text { (años) }\end{array}$ & Sexo & $\begin{array}{c}\text { Lesiones } \\
\text { Localización }\end{array}$ & $\begin{array}{c}\text { Lesiones } \\
\mathbf{n}\end{array}$ & $\begin{array}{c}\text { Evolución } \\
\text { (meses) }\end{array}$ & $\begin{array}{c}\text { Fecha del } \\
\text { diagnósico }\end{array}$ \\
\hline & & & & & & \\
1 & 02 & F & Cara & 1 & 12 & $04 / 11 / 92$ \\
2 & 08 & M & Cara & 1 & 03 & $17 / 02 / 94$ \\
3 & 09 & M & Cara & 1 & 02 & $17 / 02 / 94$ \\
4 & 01 & F & Cara & 3 & 05 & $11 / 05 / 93$ \\
5 & 57 & F & Brazo derecho & 1 & 12 & $22 / 09 / 93$ \\
6 & 13 & F & Cara & 1 & 12 & $22 / 09 / 93$ \\
7 & 13 & F & Cara & 2 & 06 & $09 / 08 / 93$ \\
8 & 02 & M & Cara & 1 & 06 & $19 / 02 / 93$ \\
9 & 02 & F & Cara & 1 & 04 & $14 / 01 / 93$ \\
10 & 07 & F & Cara & 1 & 08 & $09 / 10 / 92$ \\
11 & 10 & F & Cara & 1 & 03 & $17 / 02 / 94$ \\
12 & 05 & M & Cara & 1 & 02 & $10 / 03 / 94$ \\
13 & 03 & F & Cara & 1 & 02 & $10 / 03 / 95$ \\
14 & 04 & F & Cara & 1 & 01 & $10 / 03 / 95$ \\
\hline
\end{tabular}


3) no haber recibido tratamiento previo para leishmaniasis.

A todos los pacientes se les realizó un examen directo para estudio parasitológico y una biopsia para histopatología. En siete casos se tomaron muestras para cultivo que se enviaron a los laboratorios del CIDEIM (Centro Internacional de Investigaciones Médicas) en Cali, para tipificar las cepas causales.

El examen directo se realizó en el laboratorio de Samaniego o en el laboratorio de la División de Referencia en la ciudad de Pasto, utilizando coloración de Giemsa. Las muestras para estudio histopatológico fueron enviadas al Instituto Nacional de Salud en Santa Fé de Bogotá y al Laboratorio de Patología de la Universidad del Valle. Las muestras para cultivo y tipificación se enviaron al CIDEIM de la ciudad de Cali, siendo utilizado el medio de Seneijke. Para la tipificación, se hicieron estudios isoenzimáticos y anticuerpos monoclonales.

Para la captura e identificación de flebotomíneos, se escogió la vereda La Mesa, por haberse detectado en ésta, el mayor número de casos autóctonos de leishmaniasis. Se realizaron muestreos en diferentes épocas del año y se utilizaron varios tipos de trampas, incluyendo la de cebo humano (7) (figura 1). Se colocaron, además, trampas para roedores, buscando identificar los posibles reservorios.

\section{Resultados}

Todos los pacientes presentan lesiones clínicamente compatibles con leishmaniasis cutánea localizada. En los catorce casos estudiados, se comprobó la enfermedad por frotis y estudio histopatológico. De los siete casos enviados para cultivo, sólo tres pudieron ser tipificados, dos se encuentran inoculados en hámster (figura 9) y el resto no pudieron replicarse en cantidad suficiente. Las tres muestras aisladas correspondieron a cepas de $L$. mexicana.

Del total de pacientes, trece (93\%) fueron niños entre uno y trece años y todos presentaban lesiones localizadas en la cara (figuras 2 a 4).

La histopatología y los exámenes parasitológicos de los catorce casos estudiados mostraron un gran número de amastigotas, lo cual sugería leishmaniasis difusa (figuras 6 a 8).

Se recolectaron y disecaron 400 flebotomíneos cuya clasificación correspondió en su totalidad a Lutzomya columbiana, grupo verrucarum (figuras 11 y 12). No se encontró ningún insecto infectado en forma natural con leishmania, pero, sí se lograron infectar en los laboratorios del CIDEIM algunos especímenes de Lutzomyia columbiana a partir del hámster inoculado con la cepa de L. mexicana aislada de un paciente del presente estudio (figuras 9 a 13).

En las trampas para roedores se capturó un espécimen de Didelphis marsupiales (zarigüeya o raposa) y sus estudios en el laboratorio fueron negativos para leishmaniasis.

Todos los pacientes estudiados fueron tratados con las dosis convencionales de glucantime ${ }^{\circledR}$ (15 mg/kg/día) durante 3 a 6 semanas, con respuesta satisfactoria en la totalidad de casos (figura 5).

\section{Discusión y conclusiones}

En tres de los catorce casos se pudo tipificar la cepa responsable, que correspondía a $L$. mexicana, resultado que coincide con la tipificación del primer paciente encontrado en Samaniego en 1984 con leishmaniasis cutánea anérgica difusa.

Las características histológicas en todos los cortes sugieren el mismo comportamiento del organismo causal, encontrándose un gran número de amastigotas del parásito. Igualmente, se observó en dos casos una importante reacción de células gigantes que delimitaba las lesiones.

La identificación de las especies de flebotomíneos demostró que la totalidad de los 400 ejemplares recolectados, correspondía a Lutzomya columbiana (grupo verrucarum), hallazgo que concuerda con varios reportes anteriores de la misma región cuando, desde 1940 , se buscaba el vector de la epidemia de bartolenosis en esta zona nariñense (6) hasta 1984, cuando el INS realizó muestreos a raíz 


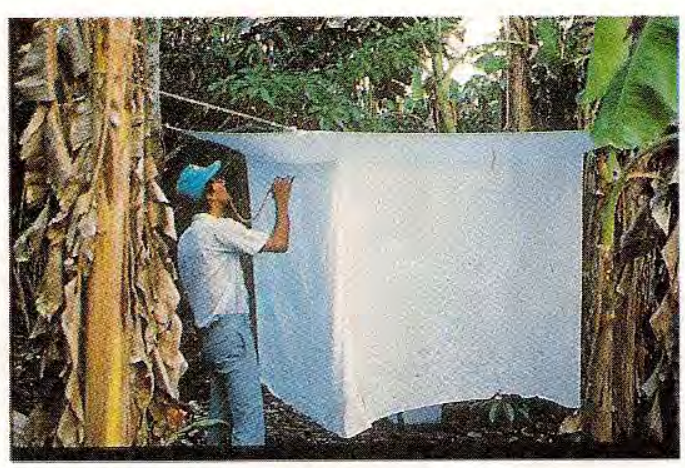

Figura 1. Trampa para capturar insectos. (Tipo Shannon).

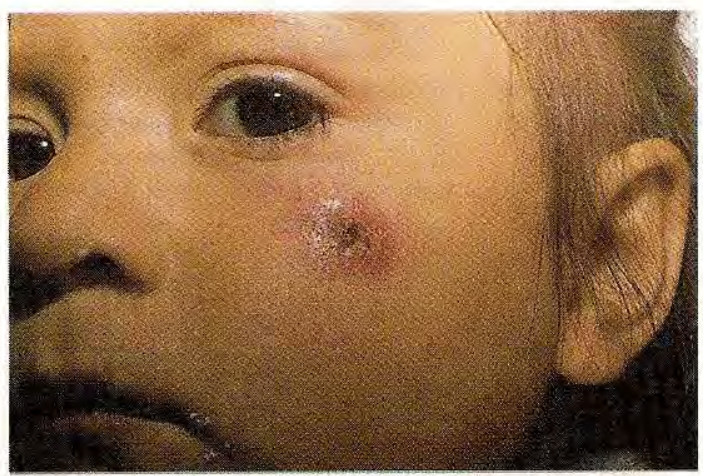

Figura 2. Lesiòn única en cara.

Aunque no logró demostrarse infección natural en los flebotomíneos capturados, sí se consiguió infectar algunos especímenes de Lutzomya columbiana expuestos a hamsters infectados con la cepa de L. mexicana aislada de un paciente procedente de Samaniego ${ }^{(8)}$.

Con este hallazgo y por evidencia epidemiológica se incrimina por primera vez a la Lutzomya columbiana como posible transmisor de L. mexicana.

El compromiso facial observado en todos los niños, podría sugerir infección intra domiciliaria durante las horas del sueño, por ser esta el área corporal más expuesta.

Contrario a lo esperado en las infecciones por cepas de L. mexicana, la respuesta de todos los pacientes al tratamiento con antimoniales (Glucantime ${ }^{\circledR}$ ) fue buena.
Debe investigarse en el futuro la posible existencia de otros focos y complementar el estudio de reservorios en dicha zona.

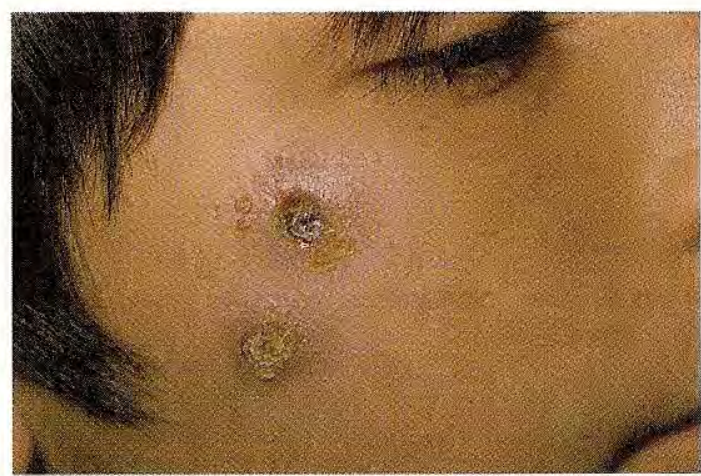

Figura 3. Lesiones múltiples en cara

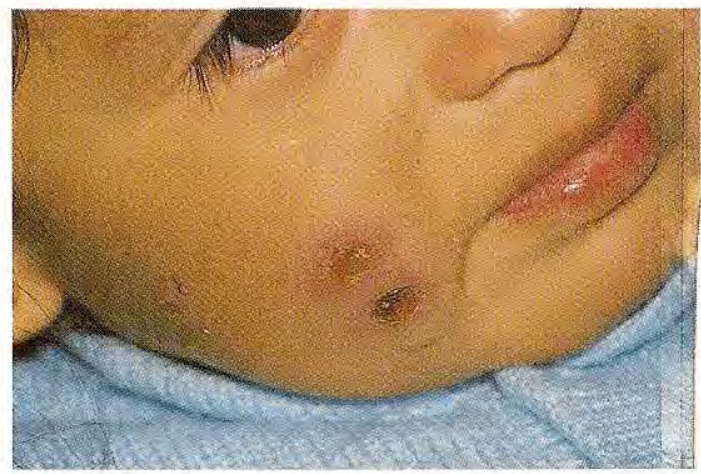

Figura 4. Lesiones múltiples en cara (Antes del tratamiento).

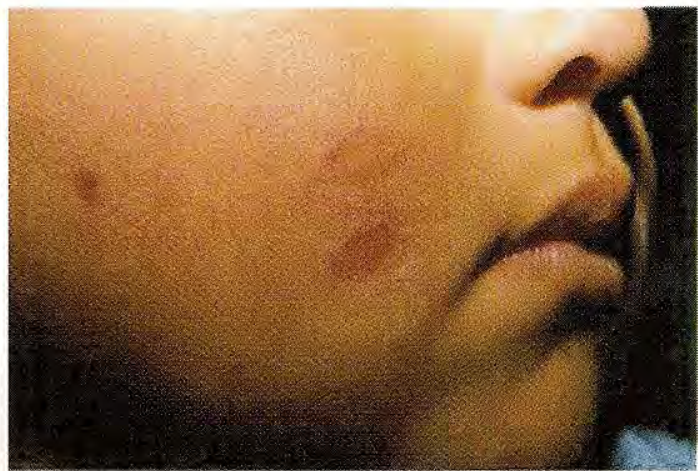

Figura 5. El mismo paciente de la Figura 4. (Después del tratamiento). 


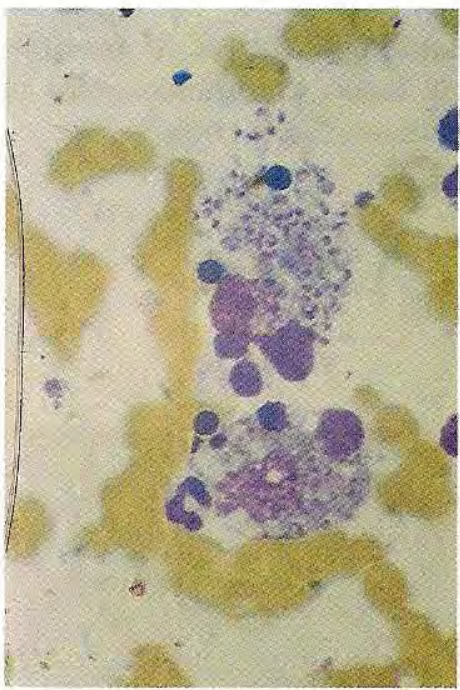

Figura 6. Frotis mostrando numerosos amostigotes (Giemsa).

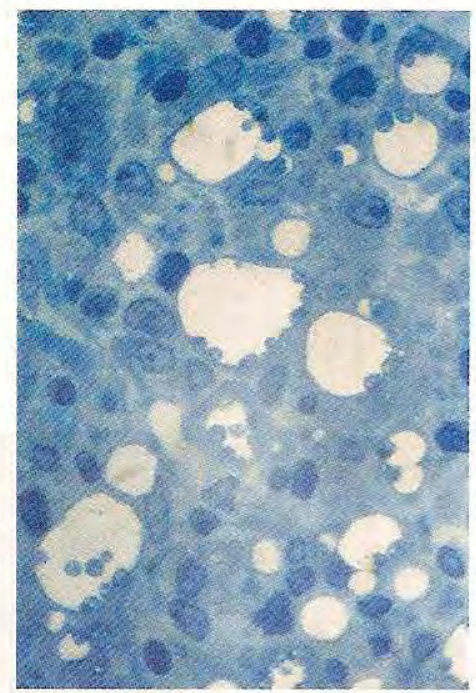

Figura 7. Corte histológico con Azul de Toluidina. Las amastigotas se observan bordeando el interior de las vacuolas.

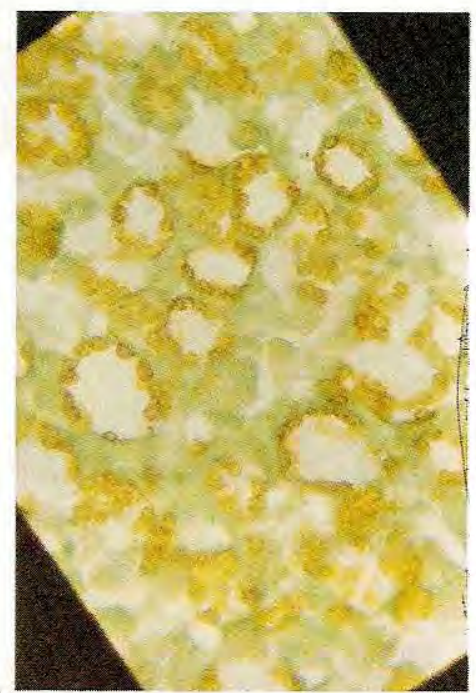

Figura 8. Preparación Inmuno-histoquímica con Avidina-biotina-peroxidasa. Toda la coloración amarillo pardusca corresponde a aglomerados de amastigotas.

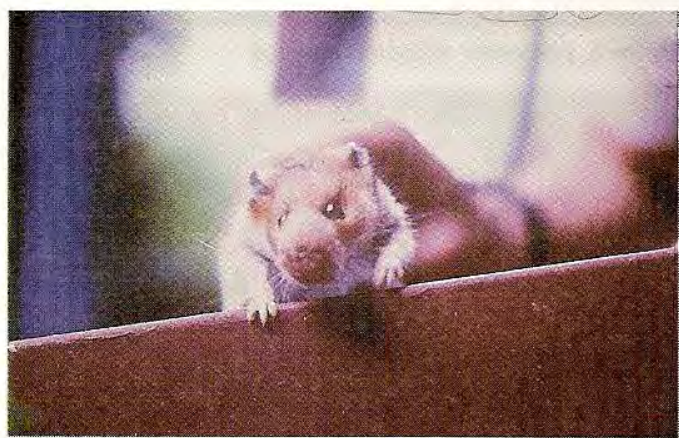

Figura 9. Infección Experimental en nariz de hamster.

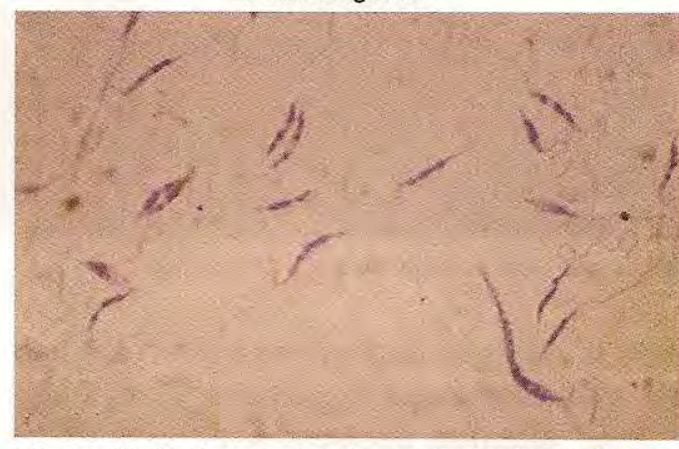

Figura 10. Cultivo mostrando promastigotas de la cepa de L. mexicana $(1000 \mathrm{x})$.

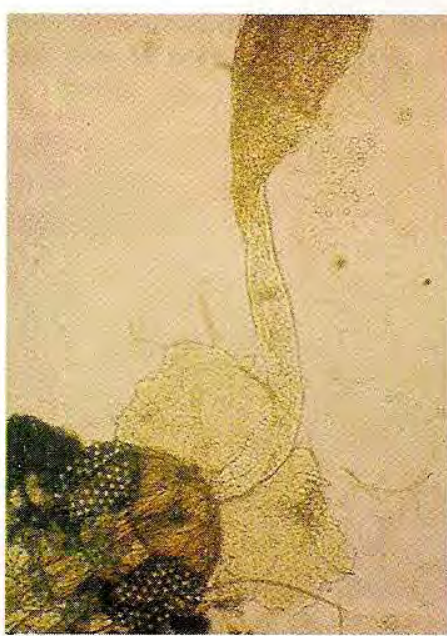

Figura 11. Disección de Lutzomya columbiana (cabeza, estructura piórica y esmodeal).

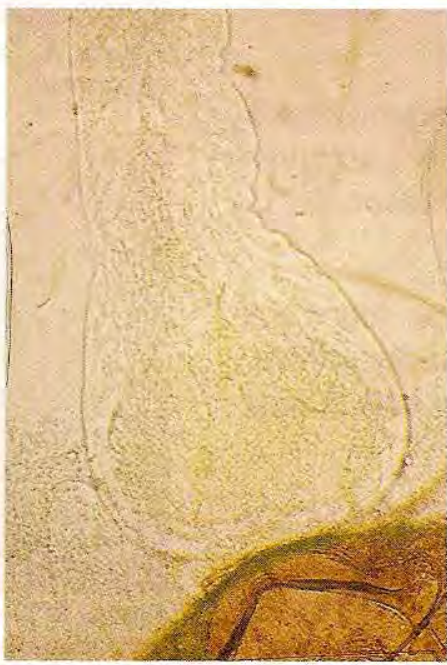

Figura 12. Válvula estomodeal mostrando desarrollo de formas infectivas.

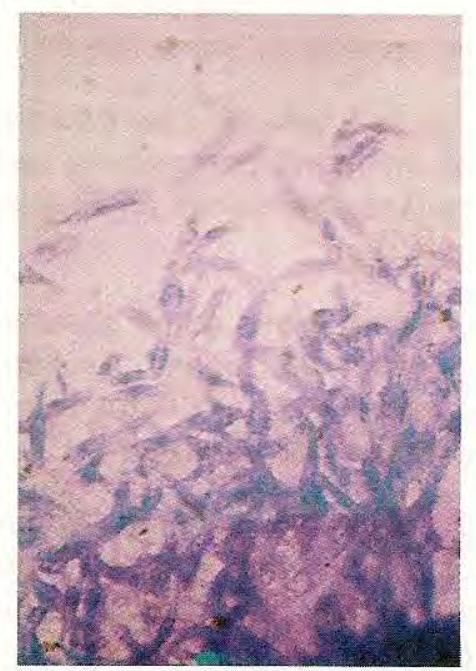

Figura 13. Ampliación de la válvula estomodeal mostrando promastigotas. 
Debe investigarse en el futuro la posible existencia de otros focos y complementar el estudio de reservorios en dicha zona.

\section{Agradecimientos}

Al doctor Gerzaín Rodríguez, patólogo del INS, por su colaboración en el procesamiento y lectura de biopsias y su aporte científico a esta publicación.

A la doctora Nancy Saravia, directora del CIDEIM, y al personal de esta entidad, quienes contribuyeron con la tipificación de la cepa y lograron la infección experimental de los vectores, parte fundamental de esta investigación.

Al doctor Luis Eduardo Bravo, patólogo de la Universidad del Valle, por sus valiosos aportes científicos y su colaboración en el procesamiento y lectura de biopsias.

A la doctora Cristina Ferro, entomóloga del INS, por su ayuda en el material bibliográfico y científico.

A la magister en ciencias biomédicas, María Clara Yépez, por su colaboración en la conformación del equipo de trabajo con los estudiantes de la Universidad de Nariño.

A los compañeros de trabajo de la División de Referencia por su colaboración y apoyo.

\section{Referencias}

1. Guzmán L, et al. Normas técnico-administrativas. Programa de prevención y control de leishmaniasis. Bogotá: Ministerio de Salud, 1992.

2. Corredor A, et al. Boletín epidemiológico, Minsalud, 1988.

3. Lainson R. Las leishmanias y las leishmaniasis del Nuevo Mundo. Bol Oficina Sanit Panam 1974; (2).

4. Rodríguez G, et al. Leishmaniasis difusa. Biomédica 1985;5(3 y 4).

5. Estrada D, et al. Identificación de especies de flebotomíneos posibles transmisores de leishmaniasis cutánea en la vereda la Mesa, municipo de Samaniego, departamento de Nariño. Pasto: Universidad de Nariño, Programa de Biología, junio de 1994.

6. Osorno E, et al. Separata. Revista de la Academia Colombiana de Ciencias Exactas, Físicas y Naturales 1972;14(53):45-68.

7. Ferro C. Informe de salidas de campo. Municipio de Samaniego, departamento de Nariño, 1985-1986.

8. Cadena $\mathbf{H}$, et al. Determinación de la competencia vectorial de Lutzomyia columbiana en la transmisión del complejo Leishmania mexicana. Resúmenes, XXII Congreso de la Sociedad Colombiana de Entomología, 1995:85.

9. Falabella $\mathbf{R}$, et al. Fundamentos de medicina.Cuarta edición. Capítulo 77, 1991. 